Article

\title{
PCB-Embedded Spiral Pattern Pick-Up Coil Current Sensor for WBG Devices
}

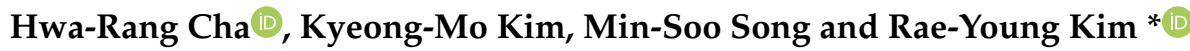 \\ Department of Electrical and Biomedical Engineering, Hanyang University, Seoul 04763, Korea; \\ hrcha33@hanyang.ac.kr (H.-R.C.); luis0898@hanyang.ac.kr (K.-M.K.); sms8171109@hanyang.ac.kr (M.-S.S.) \\ * Correspondence: rykim@hanyang.ac.kr; Tel.: +82-2-2220-2897
}

Received: 25 September 2020; Accepted: 30 October 2020; Published: 2 November 2020

\begin{abstract}
This paper proposes a current sensor for wide bandgap (WBG) devices. The current of a power semiconductor device is mainly measured using a Rogowski coil, an active current transformer, and a coaxial shunt resistor. The Rogowski coil and active current transformer are not suitable for the current measurement of surface mount WBG devices due to their operating principle. The coaxial shunt resistor causes parasitic inductance. Since WBG devices are more sensitive to parasitic inductance than silicon devices, parasitic inductance may affect circuit operation. To overcome these problems, this paper proposes a printed circuit board (PCB)-embedded spiral pattern pick-up coil current measurement for WBG devices. The proposed pick-up coil has high mutual inductance compared to the conventional pick-up coil, so the measurement sensitivity is high. In addition, there is no need for additional processing outside the PCB. Experimental results using a double pulse tester circuit are provided to verify the performance of the proposed current sensor.
\end{abstract}

Keywords: multilayer PCB pattern coil; current sensor; wide bandgap devices

\section{Introduction}

In the power converter circuit, the current measurement of power semiconductor devices is required for feedback control, fault detection, and soft switching. The current of power semiconductor devices is mainly measured using Rogowski coil, active current transformer, and coaxial shunt resistor.

Rogowski coil [1,2] is a current sensor based on electromagnetic induction. The current flowing through the conductor creates a magnetic flux. The Rogowski coil surrounds the conductor so that it is sufficiently linked with the magnetic flux. The current is estimated by applying the integrator to the voltage induced in the Rogowski coil. Because the Rogowski coil uses an air core, there is no issue due to saturation. Therefore, it has excellent linearity, provides isolation, and can measure high frequency and large current. However, the Rogowski coil is not suitable for measuring surface mount WBG devices because it must surround the conductor. In addition, in order to increase the measurement sensitivity, the number of turns of the Rogowski coil is very high, which increases the self-inductance and parasitic capacitance, thereby limiting the measurement bandwidth [3-5].

An active current transformer [6,7] is a combination of a Hall effect sensor and a current transformer (CT). CT measures AC current because it is dependent on the rate of change of magnetic flux, and Hall effect sensor measures dc and low-frequency current because it is proportional to the magnitude of the magnetic field. Therefore, the active current transformer can measure from dc to high-frequency current. However, the saturation of the core limits the current rating and the design is complicated because the two measurement methods are combined. In addition, like the Rogowski coil, it is not suitable for measuring surface mount WBG devices.

Coaxial shunt resistor [8-10] is a method of measuring current by using the voltage drop across the resistor. Because this method has a high bandwidth, it is widely used to measure the current of 
power semiconductor devices. In addition, the measurement accuracy of the high-frequency current is high because the shunt resistor hardly changes with frequency. However, parasitic inductance inevitably occurs due to the shunt resistor inserted in the circuit.

WBG devices, such as silicon carbide $(\mathrm{SiC})$ and gallium nitride $(\mathrm{GaN})$, are known to be suitable for their high efficiency and high power density, so studies have been actively conducted to apply them to power converters. However, due to the fast switching transition of WBG devices, a current sensor with a bandwidth three to five times higher than that for silicon (Si) devices and insulated-gate bipolar transistor (IGBT) devices is required for accurate current measurement [11]. That is, the current sensor having a bandwidth of several tens of $\mathrm{MHz}$ is sufficient to measure the current of Si and IGBT devices, but for the current measurement of the WBG devices, these current sensors are not suitable because of their low bandwidth. In addition, WBG devices are sensitive to parasitic inductance. In particular, in the case of $\mathrm{GaN}$ devices, parasitic inductances can cause overshoot and ringing during switching transient $[4,5,12]$. Therefore, although the bandwidth of the coaxial shunt resistor is high enough, it is not suitable for current measurement of WBG devices. Accordingly, PCB pattern-based pick-up coil current measurement methods that minimize parasitic inductance and have a wide bandwidth have been proposed [5,13-15].

In references $[5,14,15]$, a pick-up coil measurement method applying a vertical bilayer-based PCB pattern was proposed. The proposed pick-up coil is embedded in the PCB, and the current path of the device to be measure is configured outside the PCB to surround the pick-up coil. This method has the advantage of high magnetic flux linkage to the coil and high measurement sensitivity. However, since the current path is exposed to the outside, an insulator should be added between the current path and the pick-up coil, and an additional manufacturing process is required.

To overcome these problems, Wang et al. [5] proposed a method of configuring a pick-up coil next to the device to be measured. Since this method uses a single turn pick-up coil, the mutual inductance is small, and the measurement sensitivity is low. Therefore, correction is required for accurate measurement.

This paper proposes a PCB-embedded spiral pattern pick-up coil current measurement for WBG devices. The proposed pick-up coil can increase the number of turns by using the internal multiple layers of the PCB, which makes it possible to achieve high measurement sensitivity and mutual inductance. Since the current path is not exposed to the outside, no insulator is required between the pick-up coil and the current path. Therefore, this method is easy to manufacture and can reduce cost. To verify the performance of the proposed pick-up coil, a double pulse test (DPT) circuit is built. The measured current waveforms with the proposed pick-up coil and shunt resistor are compared.

\section{Consideration of the Pick-Up Coil-Based Current-Measurement Method}

\subsection{Current-Measurement Principle}

Figure 1 shows a physical model of a PCB-embedded pick-up coil. The pick-up coil consists of a PCB pattern without a core. $S$ is the closed area of the coil, $i$ is the main current flowing through the $\mathrm{PCB}$, and $v_{\text {coil }}$ is the output voltage of the coil. The main current $i$ flowing through the PCB creates a magnetic flux density $B$. The generated magnetic flux is partially linked to the pick-up coil, which is a mutual flux $\Phi$. The mutual flux $\Phi$ can be calculated as [16]

$$
\Phi=\int_{S} B \cdot d S
$$

According to Faraday's law, the magnetic flux that changes with time produces an induced electromotive force $v_{\text {ind }}$. If the self-resistance $R_{C}$ and self-inductance $L_{C}$ of the coil are neglected, 
the output voltage $v_{\text {coil }}$ of the coil becomes equal to $v_{\text {ind }}$. Further, since the induced electromotive force is proportional to the number of turns $N$ of the coil, $v_{\text {ind }}$ is expressed as follows:

$$
v_{\text {ind }}(t)=N \frac{d \Phi(t)}{d t}=M \frac{d i(t)}{d t}
$$

where $M$ is the mutual inductance. Integrating Equation (2), we have

$$
i(t)=\frac{1}{M} \int v_{\text {ind }}(t) d t .
$$

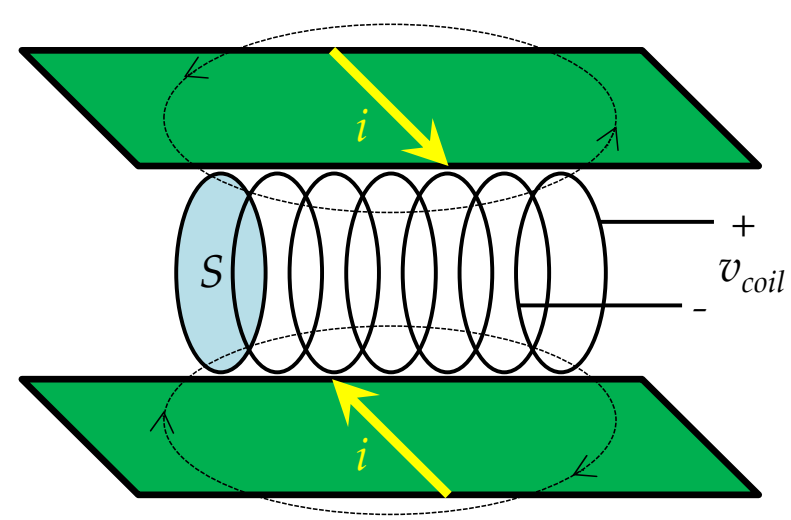

Figure 1. PCB-embedded pick-up coil structure.

Therefore, the main current $i$ flowing through PCB can be restored by integrating the coil output voltage $v_{\text {ind }}$.

\subsection{Pick-Up Coil Structure}

Figure 2 shows the coil configuration of PCB-embedded pick-up coil and its equivalent circuit. The coil is formed using two different layers on the PCB, as shown in Figure 2a, and the different layers are connected through the vias of the PCB. The pick-up coil can be represented as an equivalent circuit, as shown in Figure $2 \mathrm{~b}$, where $R_{C}, L_{C}$, and $C_{C}$ are the self-resistance, self-inductance, and self-capacitance of the pick-up coil, respectively, and $\Phi_{12}$ is the mutual flux between PCB and the pick-up coil.

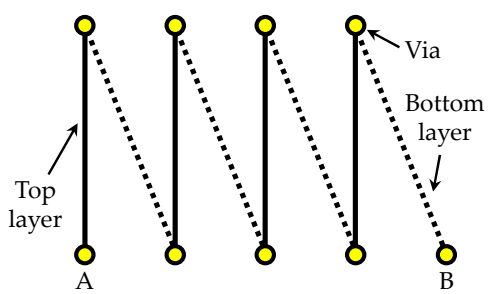

(a)

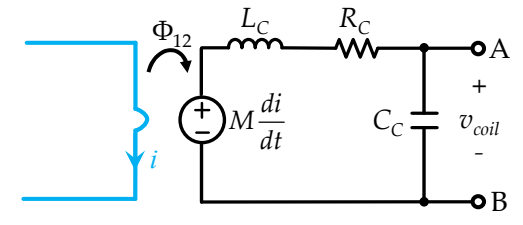

(b)

Figure 2. PCB-embedded pick-up coil: (a) coil configuration; (b) equivalent circuit.

Combining Equations (1) and (2) yields

$$
M=\frac{N \cdot B \cdot S}{i} .
$$

As shown in Equation (4), the mutual inductance $M$ is proportional to the closed area $S$ and the number of turns $N$ of the coil. Therefore, a pick-up coil with a small $S$ and $N$ has a low mutual inductance $M$. According to Equation (2), since $v_{\text {ind }}$ is proportional to $M$, the sensitivity of the current 
measurement decreases. The lower the magnitude of $v_{\text {ind }}$, the more $v_{\text {coil }}$ is affected by noise and ripple, and the accuracy of the current measurements decreases [17].

In the conventional pick-up coil, the coil pattern is arranged in the direction of the magnetic flux $\Phi_{12}$. It is difficult to increase the number of turns $N$ because the conventional coil cannot be wound inside PCB because of the double-layer coil configuration [13]. Therefore, for accurate current measurement, it is required to increase $M$ by increasing $S$ and $N$ of the pick-up coil.

\section{Proposed Spiral Pattern Pick-Up Coil}

Figure 3 shows the proposed spiral pattern pick-up coil structure. The spiral pattern pick-up coil is composed of multi-layers of PCB. The coil is configured to be wound from the outer layer to the inner layer, as shown in Figure 3a. Because the inner layer is used, $N$ can be increased without increasing the area. Therefore, the spiral pattern coil has a high mutual inductance. Figure $3 \mathrm{~b}$ shows the spiral pattern viewed from an oblique line. If the pattern starts at layer 2, as shown in Figure $3 b$, the first pattern is wound clockwise to layer 4 . The second pattern is wound from layer 4 to layer 3 , and the next pattern is wound from layer 3 to layer 4 . The fourth pattern is wound from layer 4 to layer 2 , and the four patterns are repeated.

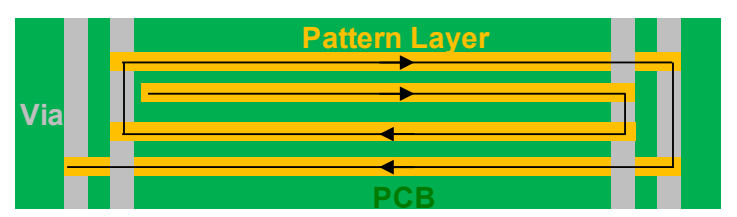

(a)

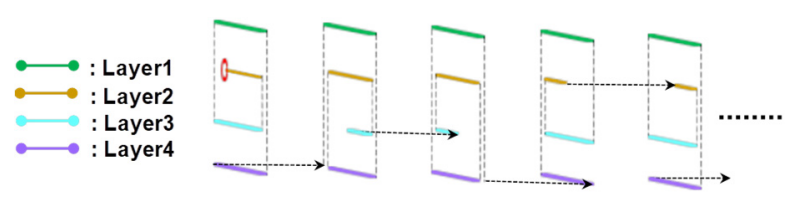

(b)

Figure 3. Spiral pattern pick-up coil PCB configuration with winding direction: (a) Cross-section view; (b) oblique view.

Figure 4 shows the finite element analysis of the pick-up coil applying the sawtooth pattern and spiral. Figure 4a shows a coil configuration using the top and bottom layers without the spiral pattern. Figure $4 \mathrm{~b}$ shows a coil configuration using the spiral pattern. The mutual inductances according to the pattern are listed in Table 1. The mutual inductance is simulated with the ANSYS Maxwell electromagnetic field simulation software. As shown in Table 1, the mutual inductance of the sawtooth pattern coil is $2.75 \mathrm{nH}$, and the mutual inductance of the spiral pattern coil is $3.10 \mathrm{nH}$. By applying the spiral pattern, the mutual inductance is improved by $12 \%$ without increasing the area.

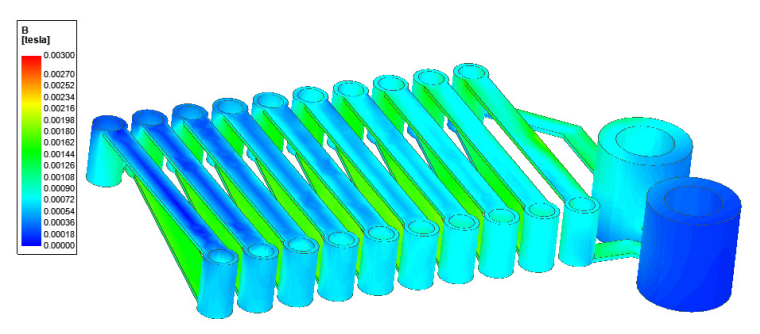

(a)

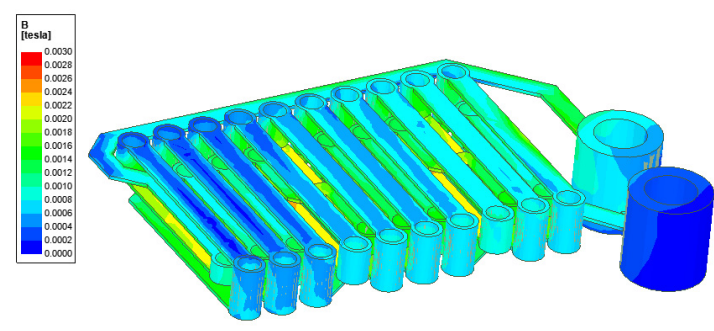

(b)

Figure 4. Configuration comparison: (a) Sawtooth pattern coil; (b) spiral pattern coil.

Table 1. Mutual inductance with respect to the pattern of the pick-up coil.

\begin{tabular}{cc}
\hline Pattern & Value (nH) \\
\hline Sawtooth pattern coil & 2.75 \\
Spiral pattern coil & 3.10 \\
\hline
\end{tabular}




\subsection{Mutual Inductance Analysis}

To achieve high measurement sensitivity by applying a spiral pattern coil, it is important to increase the mutual inductance between the measurement current and the pick-up coil through an appropriate layer selection and pattern configuration. To analyze the mutual inductance of the proposed coil, we compare the mutual inductance according to the layer selection. For comparison, a six-layer PCB board is used. The top and bottom layers are modeled to flow the main current. The coil is constructed by using inner layers (layers 1-4) excluding the top and bottom layers. First, the mutual inductance of the coil, which can be constructed using two layers among the inner layers, is analyzed. Then, the mutual inductance according to the pattern is analyzed.

\subsection{Analysis According to Layer Composition}

Figure 5a-c shows the coil structure using a pattern on two adjacent inner layers. The main current flows at the top and bottom layer. Figure 5a shows the magnetic flux density distribution according to the coil configuration using adjacent layers 1 and 2. Similarly, Figure $5 \mathrm{~b}$ shows the magnetic flux density distribution of a coil using layers 2 and 3, and layers 3 and 4, respectively.

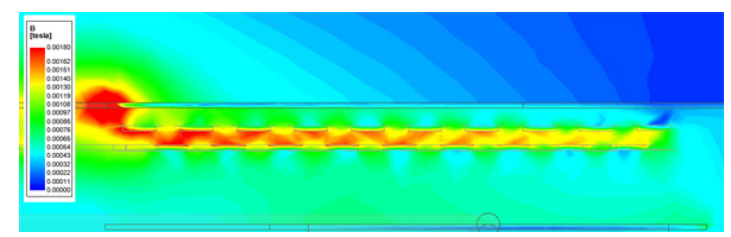

(a)

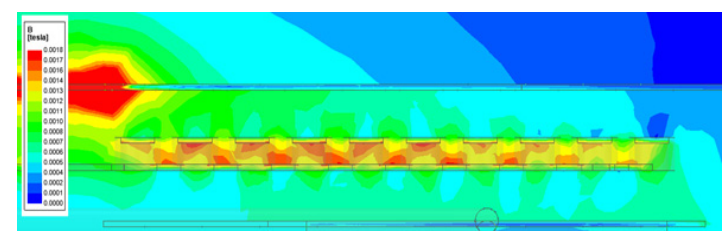

(b)

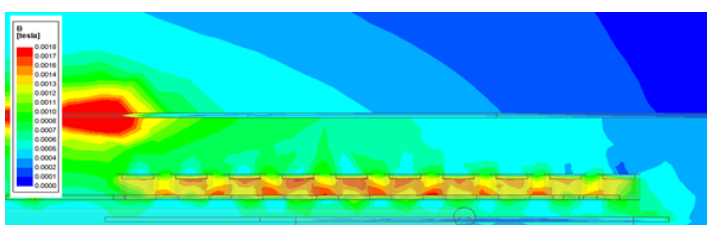

(c)

Figure 5. Finite element analysis of coil structure applied to two adjacent inner layers: (a) layers 1 and 2; (b) layers 2 and 3; and (c) layers 3 and 4.

In the bottom layer, the current flows evenly, so the color of the magnetic flux density distribution is uniform. On the other hand, since the current path of the top layer is narrowed on the left side, the magnetic flux density distribution is strong on the left side. The amount of magnetic flux linked to the closed area of the coil is highest in the coil structure of layers 2 and 3, followed by layers 1 and 2 , and layers 3 and 4 . The coil structure of layers 2 and 3 has the highest mutual inductance. The coil structure of layers 2 and 3 is arranged in the center of PCB so that magnetic flux generated by the main current flowing in the top and bottom layer is uniformly linked to the coil. However, if the coil is arranged closed to the top or bottom layer, the mutual flux with the adjacent layer increases, but the mutual flux with the opposite layer decreases greatly. Therefore, the coil structure of layers 2 and 3 has the highest mutual inductance. 
Figure 6 shows a discontinuous pattern coil, similar to the coil using two adjacent layers. Figure 6a shows the magnetic flux density distribution of the coil using layers 1 and 3 . Figure $6 \mathrm{~b}, \mathrm{c}$ shows the magnetic flux density distribution of the coil using layers 2 and 4 , and layers 1 and 4 , respectively. The coil using layers 1 and 4 has the highest mutual inductance. This is because it has the largest closed area among the three coils, and has high mutual flux with both the top and bottom layers. The simulated mutual inductances are listed in Table 2. The coil using layers 2 and 3 has the largest mutual inductance among the coil using two adjacent layers. The coil using layers 1 and 4 has the highest mutual inductance among the coil using non-adjacent layers. According to the simulation results, if the coil is arranged in the center of PCB and the area of the coil is large, the mutual inductance value is high. Hence, a multi-layer PCB pattern coil can have a higher mutual inductance as it can increase the number of turns by using the multiple inner layer.

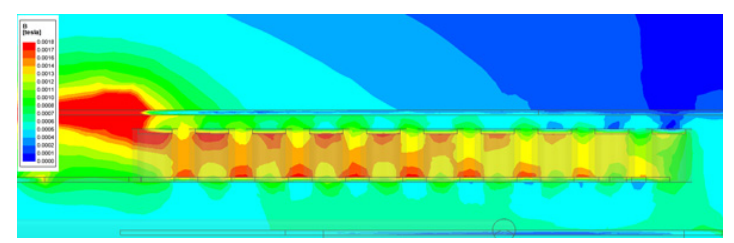

(a)

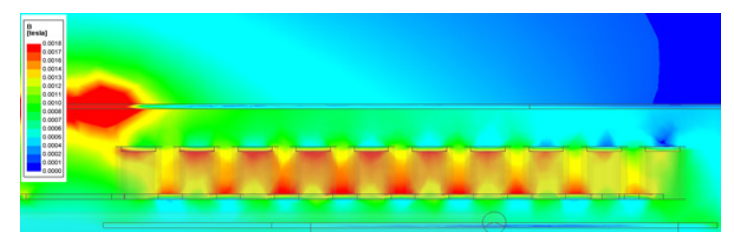

(b)

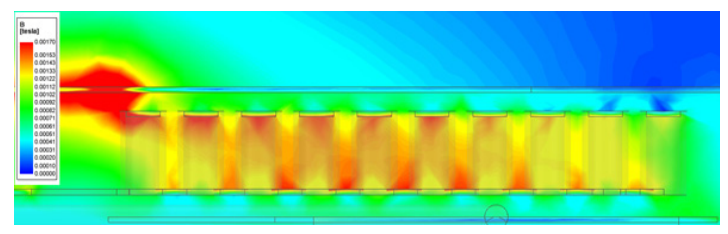

(c)

Figure 6. Finite element analysis of coil structure applied to two non-adjacent inner layers: (a) layers 1 and 3; (b) layers 2 and 4; and (c) layers 1 and 4.

Table 2. Mutual inductance according to the coil structure.

\begin{tabular}{cc}
\hline Layer Pair & Value (nH) \\
\hline Layers 1 and 2 & 0.36 \\
Layers 2 and 3 & 0.50 \\
Layers 3 and 4 & 0.39 \\
Layers 1 and 3 & 2.05 \\
Layers 2 and 4 & 1.93 \\
Layers 1 and 4 & 2.75 \\
\hline
\end{tabular}

\subsection{Analysis According to Coil Pattern Configuration}

Figure 7 shows the magnetic flux density distribution when using three different patterns applied to the spiral pattern pick-up coil. The coils are formed on the same area. The applied patterns [18] are sawtooth, triangle, and fishbone. 


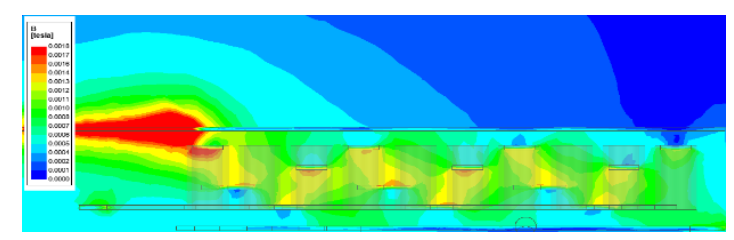

(a)

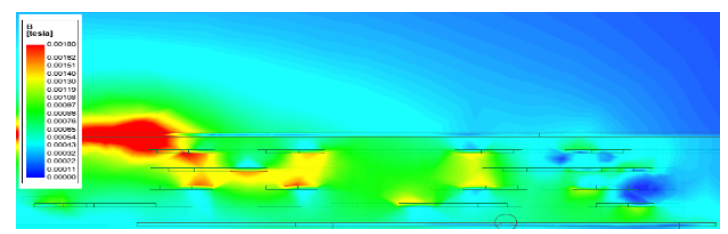

(b)

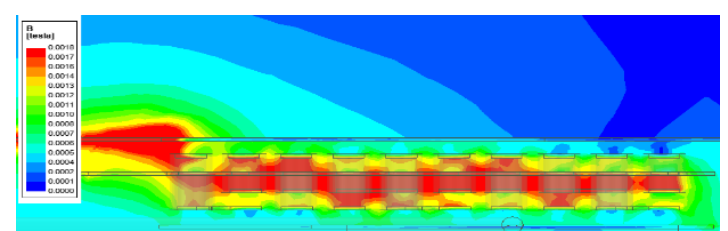

(c)

Figure 7. Finite element analysis of spiral pattern coil: (a) sawtooth pattern; (b) triangle pattern; and (c) fishbone pattern.

Figure 7a shows the magnetic flux density distribution of the pick-up coil to which the sawtooth pattern is applied. When a sawtooth pattern is applied to multi-layer, the closed area and the number of turns of the coil are reduced compare to the coil using the top and bottom layers. Therefore, the magnetic flux density distribution decreases and the mutual inductance decreases.

Figure $7 \mathrm{~b}$ shows the magnetic flux density distribution of the pick-up coil to which the triangle pattern is applied. If the triangle pattern coil is continuously wound, the winding directions of the coil are opposite each other, thereby canceling the induced voltage of the coil. Therefore, the number of turns is limited in the same area. In addition, the coil is not wound in the direction of the magnetic flux density, reducing the mutual inductance.

Figure 7c shows the magnetic flux density distribution of the pick-up coil to which the fishbone pattern is applied. When the coil is continuously wound, there is no space limitation between patterns for arranging vias on the same area. In addition, the winding direction of the coil is the same, so it has the highest number of turns. Therefore, the mutual flux is the highest and the mutual inductance is the highest.

Figure 8 shows the configuration and parameters of the coil using the sawtooth, triangle, and fishbone patterns. As discussed earlier, the fishbone pattern has the highest mutual inductance due to the high number of turns. Therefore, the fishbone pattern is most suitable for the spiral pattern pick-up coil. 


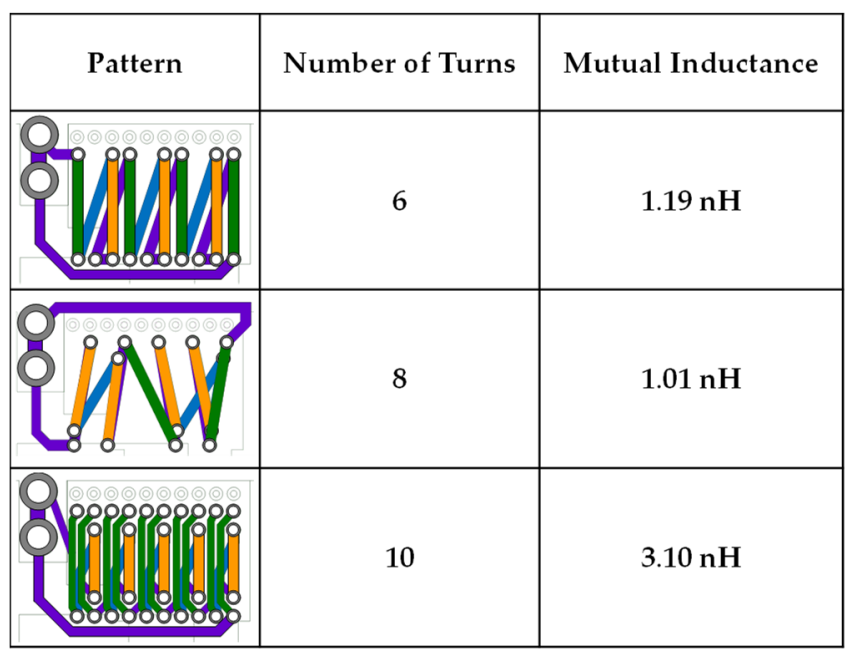

Figure 8. Physical structure of the PCB pick-up coil and the parameters.

\section{Proposed Pick-Up Coil Design and Current Path}

Figure 9 shows the design of the proposed spiral pattern pick-up coil. The proposed coil is implemented on the six-layer PCB. Because the space between the pattern and the via is limited, the winding width is chosen as $0.3 \mathrm{~mm}$. The design is created using the Altium PCB design software.

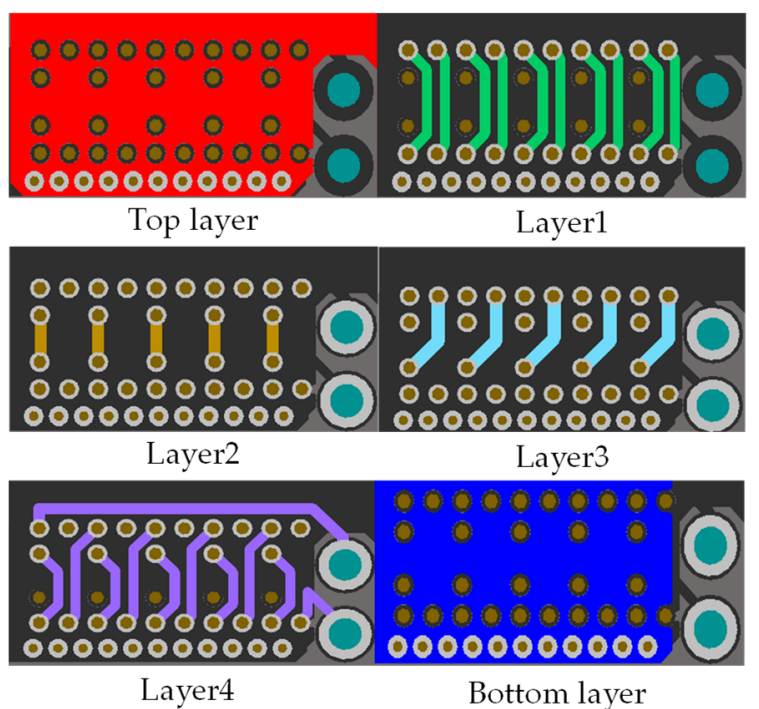

Figure 9. PCB-embedded spiral pattern pick-up coil configuration.

Figure 10 shows the conversion of a model designed with Altium software to ANSYS Q3D software to extract the parameters of the coil. The self-resistance $R_{C}$, self-capacitance $C_{C}$, and self-inductance $L_{C}$ of the proposed coil are simulated with Q3D. Through simulation, the parameters are found to be $R_{C}=0.26 \Omega, C_{C}=8.42 \mathrm{pF}$, and $L_{C}=66.29 \mathrm{nH}$, and the coil design area is $3.03 \times 6.95 \mathrm{~mm}$. 


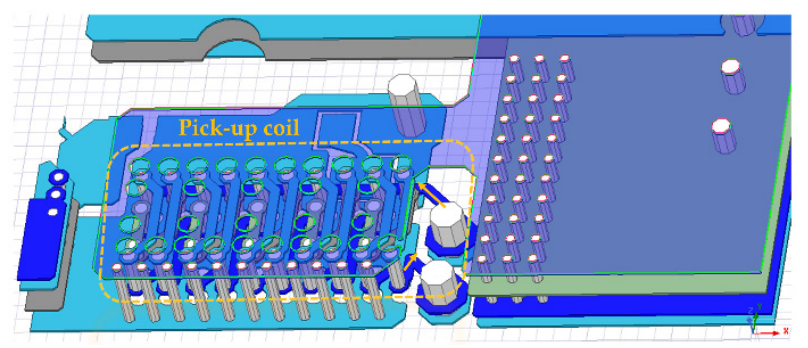

Figure 10. Q3D model of PCB with the proposed pick-up coil.

Figure 11 shows the current path for current measurement and the pick-up coil inside the PCB. As discussed earlier, the pick-up coil is implemented in the center of the PCB to increase the mutual inductance, as shown in Figure 11a. The main current path is designed to flow through the top and bottom layers where the pick-up coil is not used. And the via position is designed so that current path surrounds the pick-up coil, as shown in Figure 11b. The proposed coils are designed on inner layers 1-4. The main current path and the pick-up coil are insulated by placing clearances around the vias. Therefore, PCB-embedded pick-up coil can be manufactured without additional process work for configuring the current path.

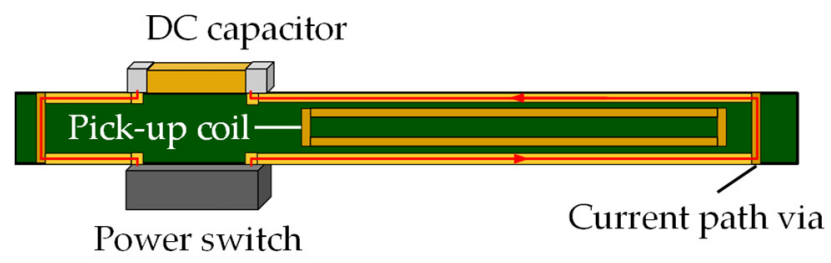

(a)

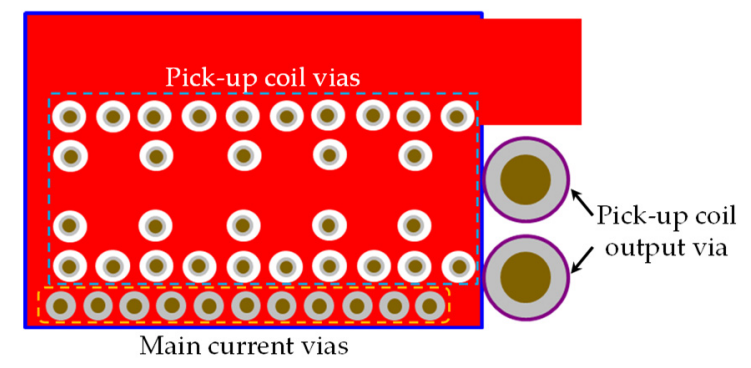

(b)

Figure 11. Structure of PCB with proposed pick-up coil: (a) side view; (b) top view.

\section{Pick-Up Coil Equivalent Model and Integration Circuit}

Figure 12 shows an equivalent circuit of the pick-up coil. $i_{S}$ represents the main switch current and $\Phi_{12}$ is the mutual flux. $L_{C}, C_{C}$, and $R_{C}$ represent the self-inductance, capacitance, and resistance of the pick-up coil, respectively. $R_{d}$ represents the damping resistance and $Z_{L}$ represents the output impedance of the coil.

The output voltage $v_{\text {coil }}$ of the pick-up coil is determined by the voltage divider of $v_{\text {ind }}$. The primary current-to-the pick-up coil output voltage transfer function is calculated as follows:

$$
G_{\text {coil }}(s)=\frac{v_{\text {coil }}(s)}{I_{s}(s)}=\frac{M Z_{L} s}{s L_{c}+R_{c}+Z_{L}} .
$$




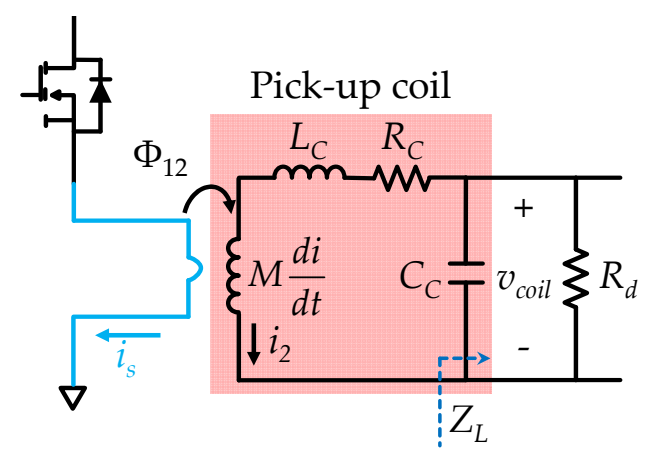

Figure 12. PCB-embedded pick-up coil equivalent circuit.

If the damping resistance $R_{d}$ is added in parallel with the self-capacitance $C_{c}$, we have

$$
Z_{L}=\frac{\frac{R_{d}}{C_{c} s}}{R_{d}+\frac{1}{C_{c} s}}=\frac{R_{d}}{R_{d} C_{c} S+1} .
$$

Substituting Equation (6) into Equation (5), we have

$$
G_{\text {coil }}(s)=\frac{\frac{M}{L_{c} C_{c}} s}{s^{2}+\left(\frac{L_{c}+R_{c} R_{d} C_{c}}{L_{c} C_{c} R_{d}}\right) s+\frac{R_{c}+R_{d}}{L_{c} C_{c} R_{d}}} .
$$

where $v_{\text {coil }}$ is a low, it is sensitive to the noise and ripple. Therefore, by adding the damping resistance $R_{d}$, it is designed as the critical damping with a damping ratio of 1 without a resonance peak. $R_{d}$ is selected as $45 \Omega$ in consideration of the parameter values extracted through Q3D and Maxwell software.

Figure 13 shows a circuit added the integrator to the pick-up coil to restore the main current. The active integrator [19] is used, which consists of the operational amplifier (op-amp), integrator resistor $R_{I N T}$, and integrator capacitor $C_{I N T}$. The voltage gain of the integrator is expressed in a low-pass filter form as follows:

$$
G_{I N T, o}(s)=\frac{1}{R_{I N T} C_{I N T} s} .
$$

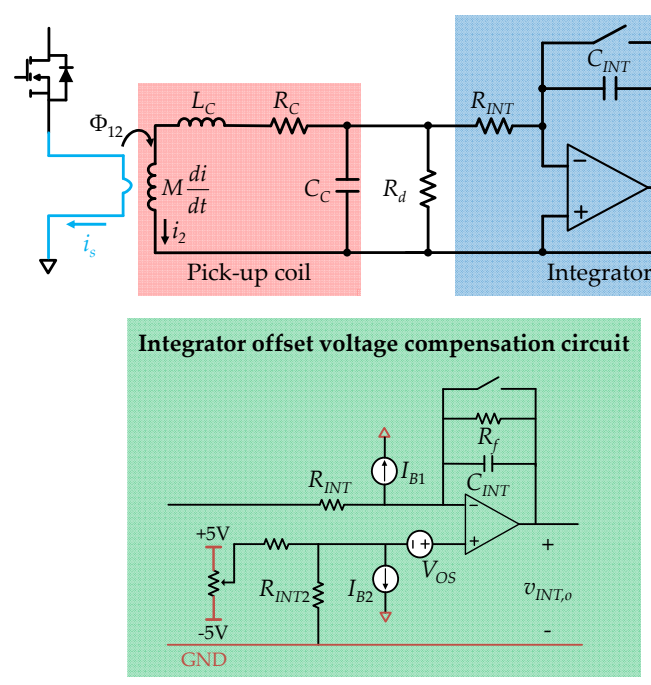

Figure 13. Integrator and offset voltage compensation equivalent circuit. 
Combining Equations (7) and (8), the main current-to-the integrator output voltage transfer function is calculated as

$$
G_{\text {sensor }}(s)=G_{\text {coil }}(s) \cdot G_{I N T, o}(s)=\frac{V_{I N T, o}(s)}{I_{s}(s)}=\frac{\frac{M}{L_{c} C_{c} R_{I N T} C_{I N T}}}{s^{2}+\left(\frac{L_{c}+R_{c} R_{d} C_{c}}{L_{c} C_{c} R_{d}}\right) s+\frac{R_{c}+R_{d}}{L_{c} C_{c} R_{d}}} .
$$

where the input signal of the integrator is 0 , the op-amp outputs an offset voltage. Therefore, to compensate for the offset voltage, the reset switch and feedback resistor $R_{f}$ are added in parallel to $C_{I N T}$ [20]. $R_{I N T 2}$ is the resistance of the non-inverting terminal, $I_{B 1}$ is the bias current of the inverting terminal, $I_{B 2}$ is the bias current of the non-inverting terminal, and $V_{O S}$ is the offset voltage. When the input signal of the integrator is 0 , the output voltage of the offset voltage compensation circuit is as follows:

$$
v_{I N T, o} \approx V_{O S} \cdot \frac{R_{f}}{R_{I N T}}+R_{f}\left(I_{B 1}-\frac{R_{I N T 2}}{R_{I N T}} \cdot I_{B 2}\right)
$$

The resistor $R_{I N T}$ and capacitor $C_{I N T}$ are selected in consideration of the bandwidth of the transfer function in Equation (9).

GaN HEMTs (GS66508T) are used as power semiconductor devices. The bandwidth of the sensor is calculated as

$$
\text { Bandwidth }=\frac{0.35}{T_{\text {edge }}} .
$$

Using Equation (11), the bandwidth of the sensor is approximately $94 \mathrm{MHz}$ [21]. The bandwidth is determined according to the rise time of the GaN HEMTs. For accurate current measurement, the sensor should have a bandwidth of $94 \mathrm{MHz}$ or more, and the bandwidth is designed to be $141 \mathrm{MHz}$.

If the gain is defined as $K$ in Equation (9), it is expressed as $R_{I N T}$ and $C_{I N T}$. Substituting $s=j \omega$ into Equation (9), $G_{\text {sensor }}$ is expressed as the standardized quadratic transfer function. Since the frequency at which the magnitude of the transfer function becomes $-3 \mathrm{~dB}$ is the bandwidth, the magnitude of the transfer function is 0.707 .

$$
\begin{gathered}
K=\frac{M}{R_{I N T} C_{I N T}} \\
G_{\text {sensor }}(s)=K \cdot \frac{1}{\left(\frac{j \omega}{\omega_{n}}\right)^{2}+\left(\frac{j \omega}{Q \omega_{n}}\right)+1} \\
\frac{K}{\sqrt{\left(1-\frac{\omega^{2}}{\omega_{n}^{2}}\right)^{2}+\left(\frac{\omega}{Q \omega_{n}}\right)^{2}}}=\frac{1}{\sqrt{2}}
\end{gathered}
$$

where $Q$ is the quality factor, $\omega_{n}$ is the natural frequency, and $\omega$ is the bandwidth frequency. $Q=0.5$, $\omega_{n}=1.34 \times 10^{9}$, and $\omega=141 \times 10^{6}$.

The sensor gain can be selected using Equation (12). In this paper, the gain is set to 0.1. Considering the fast charging and discharging of the capacitor, $R_{I N T}$ and $C_{I N T}$ are designed as $155 \Omega$ and $200 \mathrm{pF}$, respectively. Figure 14 shows the bode plot of the coil, integrator, and sensors. The bandwidth is designed to be $141 \mathrm{MHz}$. 


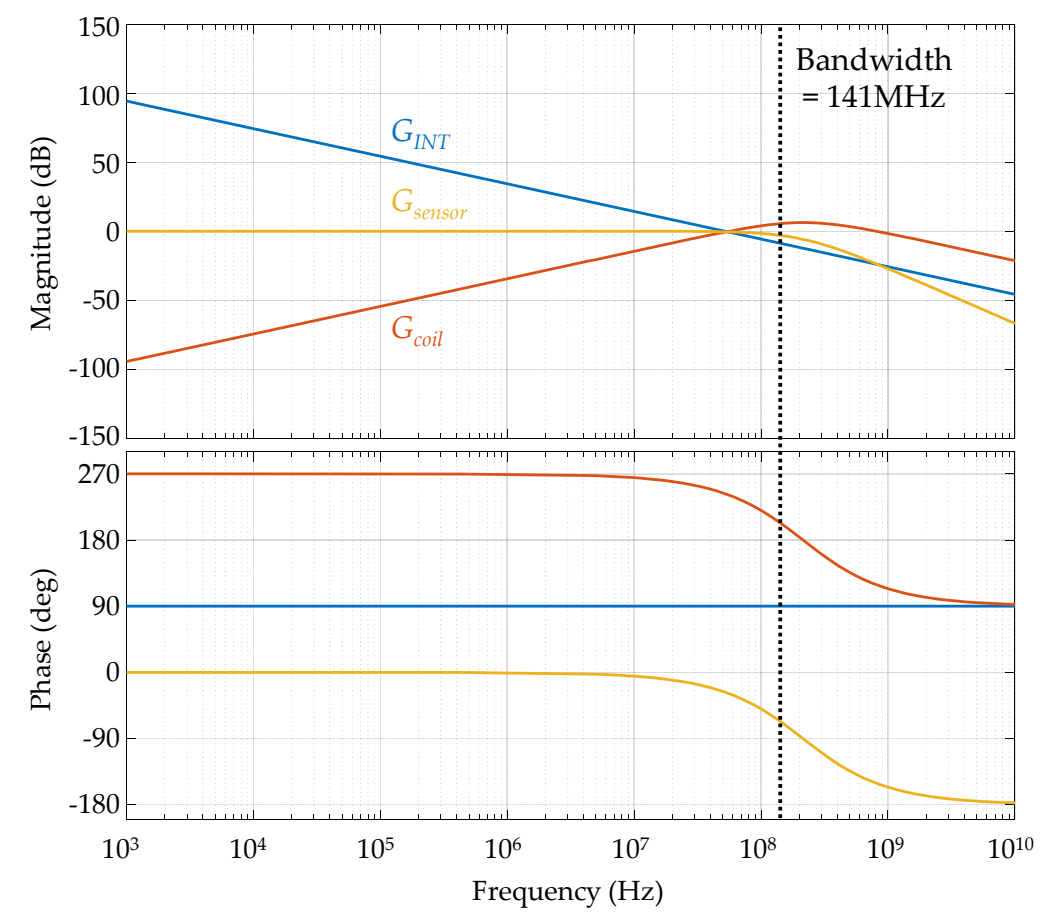

Figure 14. Magnitude and phase plot of the current-to-sensor output transfer function.

\section{Experimental Verification}

To verify the proposed method, a double-pulse test (DPT) experiment was conducted. Figure 15 shows the DPT circuit. The device under test is GaN HEMT (GS66508T). Texas Instruments OPA355 is used as an operational amplifier of the integrator. The parameter values of the pick-up coil and integrator are as specified in Table 3.

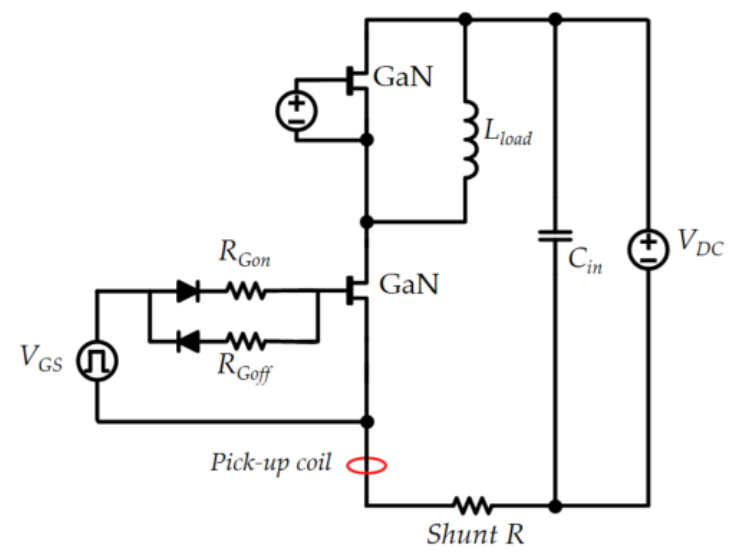

Figure 15. Double-pulse test (DPT) experiment for current measurement.

Table 3. Parameters of the pick-up coil current sensor.

\begin{tabular}{cc}
\hline Parameter & Value \\
\hline$M$ & $3.1 \mathrm{nH}$ \\
$L_{C}$ & $66.29 \mathrm{nH}$ \\
$C_{C}$ & $8.42 \mathrm{pF}$ \\
$R_{C}$ & $0.29 \Omega$ \\
$R_{d}$ & $45 \Omega$ \\
$R_{I N T}$ & $155 \Omega$ \\
$C_{I N T}$ & $200 \mathrm{pF}$ \\
\hline
\end{tabular}


To verify whether the proposed spiral pattern pick-up coil satisfies the bandwidth required for measuring the current of WBG HEMTs, a coaxial shunt resistor (SDN-414-10) was used for comparison. Because the shunt resistor does not change its resistance according to the frequency and has a high bandwidth, it satisfies the bandwidth required for measuring the current of a WBG device. Thus, it can be said that the shunt resistor can measure the current of the WBG device most accurately. Therefore, the current measurement performance is verified by comparing the waveform measured with the shunt resistor and the waveform measured with the pick-up coil. The integrator gain of the pick-up coil was selected to be equal to the shunt resistance, through Equation (12).

The DPT was conducted under the experimental conditions listed in Table 4. The specifications of the shunt resistor and the design parameters of the pick-up coil are listed in Table 4 . The first pulse is injected for $5.5 \mu \mathrm{s}$ and the second pulse is injected for $2 \mu \mathrm{s}$. To compare the performance of the two current sensors, the current was measured simultaneously, and the two waveforms were compared.

Table 4. DPT test conditions for each current sensor.

\begin{tabular}{ccc}
\hline Parameter & Shunt Resistor & Pick-Up Coil \\
\hline DC voltage & $400 \mathrm{~V}$ & $400 \mathrm{~V}$ \\
Test current & $10 \mathrm{~A}$ & $10 \mathrm{~A}$ \\
Gain & 0.1 & 0.1 \\
Bandwidth & $2 \mathrm{GHz}$ & $141 \mathrm{MHz}$ \\
\hline
\end{tabular}

Figure 16 shows the output values of the shunt resistor and the proposed pick-up coil. It can be seen that the waveforms of the two current sensors are almost identical in all sections of the DPT experiment. If the offset of the actual op-amp is not compensated, the integral value due to the offset changes. Then, the rise slope of the current in the DPT waveform would be different. It can be seen indirectly that the offset compensation circuit and the reset switch are well applied.

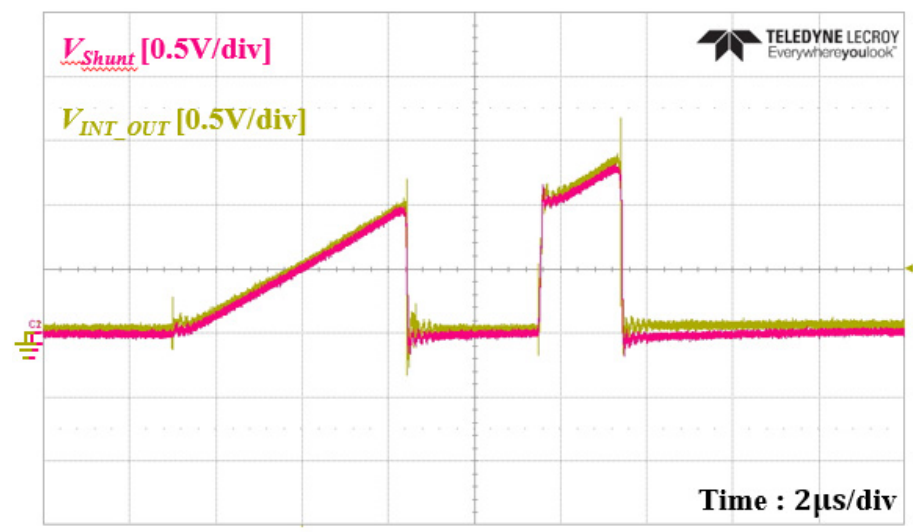

Figure 16. Overall DPT waveform of the shunt resistor and spiral pick-up coil.

Figure 17 shows the enlarged waveform of the transient section in which the switch turns off and on in the DPT experiment. Figure 17a shows the waveform when the switch current turns off at $10 \mathrm{~A}$, and Figure $17 \mathrm{~b}$ shows the waveform when the switch current turns on at $10 \mathrm{~A}$. The two waveforms in Figure 17 indicate that the current sensor using the pick-up coil tracks the shunt resistance well in the transient section, as well as over the entire DPT waveform. As shown in Figure 17a, the falling time of the current is almost the same as that of the shunt resistor. In Figure 17b, the rise time and peak value of the current versus the shunt resistor are almost the same. 


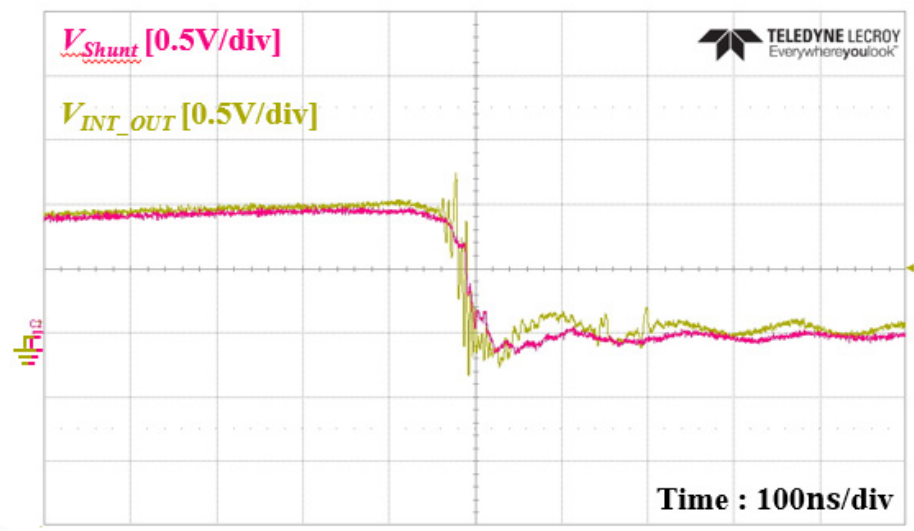

(a)

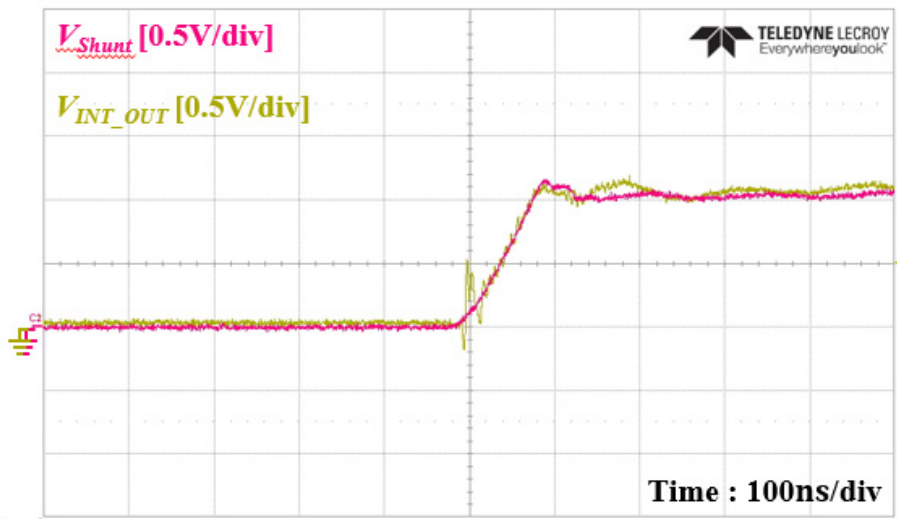

(b)

Figure 17. Transient DPT waveform of a shunt resistor and a spiral pick-up coil: (a) turn-off; (b) turn-on.

Unfortunately, both waveforms can be seen to generate ripples, due to noise components. The pick-up coil can theoretically be called an isolated current sensor independent of the power loop. However, a coupling capacitor can be seen between the pick-up coil and the power loop. Consequently, a noise current is generated by the coupling capacitor in the transient section and a ripple is formed in the integrator output, as shown in Figure 17. Coupling capacitors are also an important factor when designing a pick-up coil and should be considered sufficiently during the design phase.

\section{Conclusions}

In this paper, we proposed a PCB-embedded spiral pattern pick-up coil that has high mutual inductance when comparing PCB layer structures and applying patterns. When the proposed PCB-embedded spiral pattern pick-up coil was used, the size of the current measurement sensor was reduced. Moreover, it had a high mutual inductance, thereby increasing the current measurement sensitivity and accuracy. The proposed coil was proved to follow the switch current almost identically in the entire section and the transient section, compared to a shunt resistor with high bandwidth.

Author Contributions: Conceptualization, K.-M.K. and R.-Y.K.; formal analysis, K.-M.K. and H.-R.C.; project administration, R.-Y.K.; supervision, R.-Y.K.; validation, H.-R.C. and M.-S.S.; visualization, K.-M.K. and R.-Y.K.; writing—original draft, K.-M.K.; writing—review and editing, H.-R.C., M.-S.S., and R.-Y.K. All authors have read and agreed to the published version of the manuscript.

Funding: This work was supported by "Human Resources Program in Energy Technology" of the Korea Institute of Energy Technology Evaluation and Planning (KETEP), granted financial resource from the Ministry of Trade, Industry \& Energy, Republic of Korea (No. 2018201010650A, No. 20184010201710).

Conflicts of Interest: The authors declare no conflict of interest. 


\section{References}

1. Abdi-Jalebi, E.; McMahon, R. Simple and Practical Construction of High-Performance, Low-Cost Rogowski Transducers and Accompanying Circuitry for Research Applications. In Proceedings of the IEEE Instrumentation and Measurement Technology Conference, Ottawa, ON, Canada, 16-19 May 2005; pp. 354-358.

2. Ray, W.; Hewson, C. High performance Rogowski current transducers. In Proceedings of the Conference Record of the 2000 IEEE Industry Applications Conference. Thirty-Fifth IAS Annual Meeting and World Conference on Industrial Applications of Electrical Energy (Cat. No.00CH37129), Rome, Italy, 8-12 October 2002.

3. Yang, C.; Pei, Y.; Wang, L.; Yu, L.; Zhao, C.; Zhu, M.; Liu, X. Analysis and design of a low-cost well-performance and easy-to-design current sensing circuit suitable for SiC MOSFETs. IEEE Trans. Power Electron. 2020, 35, 13356-13366. [CrossRef]

4. Li, K.; Videt, A.; Idir, N. Using current surface probe to measure the current of the fast power Semiconductors. IEEE Trans. Power Electron. 2014, 30, 2911-2917. [CrossRef]

5. Wang, K.; Yang, X.; Li, H.; Wang, L.; Jain, P. A High-bandwidth integrated current measurement for detecting switching current of fast GaN devices. IEEE Trans. Power Electron. 2018, 33, 6199-6210. [CrossRef]

6. Poulichet, P.; Costa, F.; Labouré, É. A new high-current large bandwidth DC active current probe for power electronics measurements. IEEE Trans. Ind. Electron. 2005, 52, 243-254. [CrossRef]

7. Dalessandro, L.; Karrer, N.; Kolar, J.W. High-performance planar isolated current sensor for power electronics applications. IEEE Trans. Power Electron. 2007, 22, 1682-1692. [CrossRef]

8. Lind, K.; SØrsdal, T.; Slinde, H. Design, modeling, and verification of high-performance AC-DC current shunts from inexpensive components. IEEE Trans. Instrum. Meas. 2008, 57, 176-181. [CrossRef]

9. Filipski, P.S.; Boecker, M. AC-DC current shunts and system for extended current and frequency ranges. IEEE Trans. Instrum. Meas. 2006, 55, 1222-1227. [CrossRef]

10. Johnson, C.M.; Palmer, P.R. Current measurement using compensated coaxial shunts. IEEE Proc. Sci. Meas. Technol. 1994, 141, 471-480. [CrossRef]

11. ABCs of Probes. Available online: http://info.tek.com/www-abcs-of-probes-primer.html (accessed on 16 January 2016).

12. Gareau, J.; Hou, R.; Emadi, A. Review of loss distribution, analysis, and measurement techniques for GaN HEMTs. IEEE Trans. Power Electron. 2020, 35, 7405-7418. [CrossRef]

13. Zhao, L.; van Wyk, J.D.; Odendaal, W.G. Planar Embedded Pick-up Coil Sensor for Integrated Power Electronic Modules. In Proceedings of the Nineteenth Annual IEEE Applied Power Electronics Conference and Exposition, Anaheim, CA, USA, 22-26 February 2004; pp. 945-951.

14. Xue, Y.; Lu, J.; Wang, Z.; Tolbert, L.M.; Blalock, B.J.; Wang, F. A Compact Planar Rogowski Coil Current Sensor for Active Current Balancing of Parallel-Connected Silicon Carbide MOSFETs. In Proceedings of the IEEE Energy Conversion Congress and Exposition (ECCE), Pittsburgh, PA, USA, 14-18 September 2014; pp. 4685-4690.

15. Kuwabara, Y.; Wada, K.; Guichon, J.-M.; Schanen, J.-L.; Roudet, J. Implementation and Performance of a Current Sensor for a Laminated Bus Bar. In Proceedings of the IEEE Energy Conversion Congress and Exposition (ECCE), Milwaukee, WI, USA, 18-22 September 2016; pp. 1-7.

16. Cederlof, M. Inductive Charging of Electrical Vehicles: System Study. Master's Thesis, KTH Electrical Engineering, Stockholm, Sweden, 2012.

17. Wang, J.; Shen, Z.; Burgos, R.; Boroyevich, D. Design of a high-bandwidth Rogowski current sensor for gate-drive short-circuit protection of $1.7 \mathrm{kV}$ SiC MOSFET power modules. In Proceedings of the 3rd IEEE Workshop on Wide Bandgap Power Devices and Applications (WiPDA), Blacksburg, VA, USA, 2-4 November 2015; pp. 104-107.

18. Tsukuda, M.; Koga, M.; Nakashima, K.; Omura, I. Micro PCB Rogowski coil for current monitoring and protection of high voltage power modules. Microelectron. Reliab. 2016, 64, 479-483. [CrossRef]

19. Li, W.; Yin, X.; Chen, D.; Chen, W.; Zhang, Z.; Xia, Y. The Study of Transient Performance of Current Sensor Based on Rogowski Coil and Its Application in Dynamic Simulation Experiment. In Proceedings of the International Conference on Power System Technology, Chongqing, China, 22-26 October 2006. 
20. Wang, J.; Shen, Z.; Burgos, R.; Boroyevich, D. Integrated switch current sensor for short-circuit protection and current control of $1.7 \mathrm{kV} \mathrm{SiC} \mathrm{MOSFET} \mathrm{modules.} \mathrm{In} \mathrm{Proceedings} \mathrm{of} \mathrm{the} \mathrm{IEEE} \mathrm{Energy} \mathrm{Conversion} \mathrm{Congress}$ and Exposition (ECCE), Milwaukee, WI, USA, 18-22 September 2016; pp. 1-7.

21. Lidow, A.; De Rooij, M.; Strydom, J.; Reusch, D.; Glaser, J. GaN Transistors for Efficient Power Conversion; John Wiley \& Sons Inc.: Piscataway, NJ, USA, 2014; pp. 83-85.

Publisher's Note: MDPI stays neutral with regard to jurisdictional claims in published maps and institutional affiliations.

(C) 2020 by the authors. Licensee MDPI, Basel, Switzerland. This article is an open access article distributed under the terms and conditions of the Creative Commons Attribution (CC BY) license (http://creativecommons.org/licenses/by/4.0/). 